\title{
NON GAUSSIAN RESPONSE OF BRIDGES SUBJECTED TO TURBULENT WIND - EFFECT OF THE NON LINEARITY OF AERODYNAMIC COEFFICIENTS
}

\author{
Vincent Denoël ${ }^{1}$, Hervé Degée ${ }^{1}$ \\ ${ }^{1}$ University of Liège, Department of mechanics of Materials and Structures \\ e-mail: v.denoel@ulg.ac.be, h.degee@ulg.ac.be
}

Keywords: Wind engineering, Non Gaussianity, Quasi-steady wind loading, aerodynamic coefficients, turbulence, Viaduct of Millau

\begin{abstract}
In the context of a non linear quasi-steady wind loading, this paper shows the importance of the non linearity of the wind loading. More particularly, it focuses of the influence, on the structural response, of the non linearity of the aerodynamic coefficients. It is shown that, in case of 2-D turbulent flow, the influence of this kind of non linearity may be significant. To do so, statistical characteristics of the loading as well as those of the response are computed in such a turbulent flow.
\end{abstract}




\section{INTRODUCTION}

Since the early developments in wind engineering, the aerodynamic loading has been linearized. The primary motivations were mainly the limitation of computation means, which nowadays have no more reason to be. These last decades, the very first developments of Davenport [1] have been upgraded in order to account for more complex phenomena. Many researches have been dealing with some of them like flutter or galloping. It is quite amazing to see that they are still represented by linear wind loading models [2].

In this paper, we go back to the very first developments of Davenport, avoid the assumptions of linearization and see how a non linear wind loading model can bring new observations. A more precise attention is devoted to the influence of the non linearity of aerodynamic coefficients with respect to the angle of attack.

As a first step, the quasi-static wind loading model is presented and a non linear (quadratic) expression is extracted from the complete non linear relation. The statistical characterization of the loading is then presented in an analytical way, and checked by Monte Carlo simulation. At this stage, the importance of the non linearity of the aerodynamic coefficient can already be assessed.

A second part recalls briefly how to solve the non Gaussian dynamic problem and presents a simplified procedure to estimate the effects of the non linearity of an aerodynamic coefficient on the structural response. An example of illustration is given with the drag coefficient of the Viaduct of Millau (France).

\section{QUASI-STEADY WIND MODEL}

Forces acting on a body immerged in a fluid result from the normal pressures acting on it. In civil engineering applications, and more particularly in bridge engineering, three forces (drag, lift and moment) are generally considered. For example, the aerodynamic drag force $F_{D}$ acting on a fixed body in a uniform flow with constant velocity $V$ can be expressed by [3]:

$$
F_{D}=\frac{1}{2} C_{D} \rho B V^{2}
$$

where $\rho$ and $B$ represent respectively the air density and the width of the body, i.e. the bridge deck in the further application. Even if CFD models can simulate the flow around a bluff body, aerodynamic coefficients $\left(C_{D}, C_{L}\right.$ and $\left.C_{M}\right)$ are generally estimated by means of wind tunnel measurements. For usual applications, these coefficients exhibit a significantly non linear dependency with respect to the wind angle of attack $i$, i.e. the angle between the wind direction and the bridge deck (see Fig. 1-a).

(a)
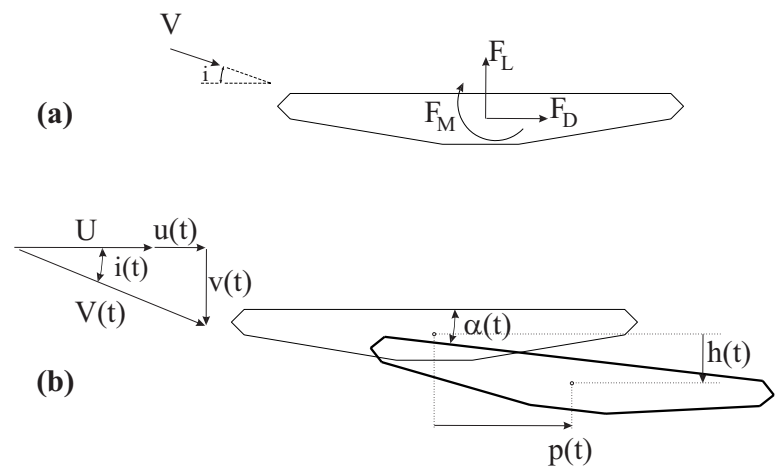

Fig. 1. (a) Aerodynamic forces in a uniform flow ; (b) Components of turbulence and displacements of the deck 
Civil engineering structures are built in the atmospheric boundary layer. In this region the wind flow is known to be turbulent. It is composed of a mean velocity $U$ and longitudinal $u(t)$ and transverse $v(t)$ fluctuations which are generally modelled as Gaussian stochastic processes [4]. Davenport [1] proposed to express the forces acting on a structure immerged in such a turbulent flow by the same relation:

$$
F_{D}(t)=\frac{1}{2} C_{D}[i(t)] \rho B V^{2}(t)
$$

where both the wind angle of attack $i(t)$ and the squared wind velocity $V^{2}(t)$ are now time-dependent. Based on geometric relations, with notations of Fig. 1-b, these two quantities can be expressed as a function of the bridge motion $(h, p, \alpha)$ and the components of the turbulence:

$$
\begin{aligned}
& i(t)=\operatorname{ArcTan}\left(\frac{v(t)-\dot{h}(t)}{U+u(t)-\dot{p}(t)}\right)-\alpha(t) \\
& V^{2}(t)=(U+u(t)-\dot{p}(t))^{2}+(v(t)-\dot{h}(t))^{2}
\end{aligned}
$$

Because of the non linearity of the aerodynamic coefficients (with respect to the angle of attack) and because of the non linearity of relations (3), equation (2) shows that the quasisteady aerodynamic forces are non linear functions of Gaussian processes.

In the atmospheric boundary layer, the usual components of turbulence $u(t)$ and $v(t)$ are smaller than the mean wind velocity $U$. Because of serviceability limitations, the structural velocities are also much smaller than the mean wind velocity. This indicates that a Taylor series expansion can be derived for the expression of the loading.

By introducing the Taylor series expansion of the aerodynamic coefficient:

$$
C_{D}(i)=c_{0}+i c_{1}+\frac{i^{2}}{2 !} c_{2}+\frac{i^{3}}{3 !} c_{3}+\ldots
$$

and the same kind of development for the non linear relations of Equ (3), equation (2) can be written [5]:

$$
\frac{F_{D}(t)}{\frac{1}{2} \rho B U^{2}}=F_{s e}(t)+\sum_{l=0}^{\infty} \sum_{k=0}^{\infty} F_{k l} \frac{u^{l}(t) v^{k}(t)}{U^{k+l}}
$$

where $F_{\text {se }}(t)$ is a non linear expression of time-dependent self-excited forces (terms depending on the structural motion) and parametric forces (cross-terms between turbulence components and structural velocity). It is known [2, 3] that the expressions of the self-excited forces obtained with a quasi-steady approach do not represent precisely the interaction between the structure and the flow. For this reason, in the following, buffeting forces (including the mean force) will be considered only.

The double summation contains the mean wind force $(k=0, l=0)$ as well as the non linear buffeting forces. Table 1 gives the first $F_{k l}$ coefficients. It can be seen that the order $n$ coefficient of the aerodynamic force $\left(c_{n}\right)$ doesn't affect the forces coefficients lower than $n$. For example, $c_{2}$ appears in the expressions of coefficients $F_{k l}$ for which $k+l \geq 2$. This means that the second order is thus the minimum order of the loading to be considered in order to show the influence of the non linearity of aerodynamic coefficients. 


\begin{tabular}{lllll}
\hline$F_{k l}$ & $k=0$ & $k=1$ & $k=2$ & $k=3$ \\
\hline$l=0$ & $c_{0}$ & $c_{1}$ & $c_{0}+c_{2} / 2$ & $2 / 3 c_{1}+c_{3} / 6$ \\
$l=1$ & $2 c_{0}$ & $c_{1}$ & 0 & $-2 / 3 c_{1}-c_{3} / 6$ \\
$l=2$ & $c_{0}$ & 0 & 0 & $2 / 3 c_{1}+c_{3} / 6$ \\
$l=3$ & 0 & 0 & 0 & $-2 / 3 c_{1}-c_{3} / 6$ \\
\hline
\end{tabular}

Table 1. Coefficients of the Taylor series expansion for the non linear buffeting loading model.

The complete non linear expression of the loading can't be studied in an analytical way. In the following, three different loadings only will be considered:

- the first one consists in realizing the Taylor series up to the second order terms (to show the influence of the non linearity of the aerodynamic coefficient $c_{2}$ ):

$$
\frac{F_{D}(t)}{\frac{1}{2} \rho B U^{2}}=c_{0}+2 c_{0} \frac{u(t)}{U}+c_{1} \frac{v(t)}{U}+c_{0} \frac{u^{2}(t)}{U^{2}}+c_{1} \frac{u(t) v(t)}{U^{2}}+\left(c_{0}+\frac{c_{2}}{2}\right) \frac{v^{2}(t)}{U^{2}}
$$

- in order to estimate the effects of the non linearity of the aerodynamic coefficient, a second loading can be considered by adding the assumption of linear aerodynamic coefficient $\left(c_{2}=0\right)$ :

$$
\frac{F_{D}(t)}{\frac{1}{2} \rho B U^{2}}=c_{0}+2 c_{0} \frac{u(t)}{U}+c_{1} \frac{v(t)}{U}+c_{0} \frac{u^{2}(t)}{U^{2}}+c_{1} \frac{u(t) v(t)}{U^{2}}+c_{0} \frac{v^{2}(t)}{U^{2}}
$$

- the linear approximation adopted by Davenport (which is also frequently used for bridge designs):

$$
\frac{F_{D}(t)}{\frac{1}{2} \rho B U^{2}}=c_{0}+2 c_{0} \frac{u(t)}{U}+c_{1} \frac{v(t)}{U}
$$

\section{NON GAUSSIANITY OF THE WIND LOADING}

\subsection{Analytical developments}

In this latter expression, the aerodynamic force is expressed as a linear combination of Gaussian processes. It is thus also a Gaussian process. This property led to the famousness of this linearization. Indeed, in the design procedure, it is convenient to work with Gaussian processes, simply because two statistical values, the mean and the standard deviation, are sufficient to characterize them fully. They are also quite attractive because the statistics of extreme values (useful for the design) can be obtained in closed forms [1, 6, 7].

Some analytical developments will be presented in the following. They will be illustrated with the expression of the drag force of the Viaduct of Millau. The measured drag coefficient is represented in figure 2 as a function of the wind angle of attack. Two approximations, a linear one and a quadratic one, are also represented. They are obtained by least-square fitting with a non uniform distribution [5]. The parameters corresponding to the quadratic approximation are:

$$
c_{0}=0.0830 ; c_{1}=0.2020 ; c_{2}=-3.3999
$$




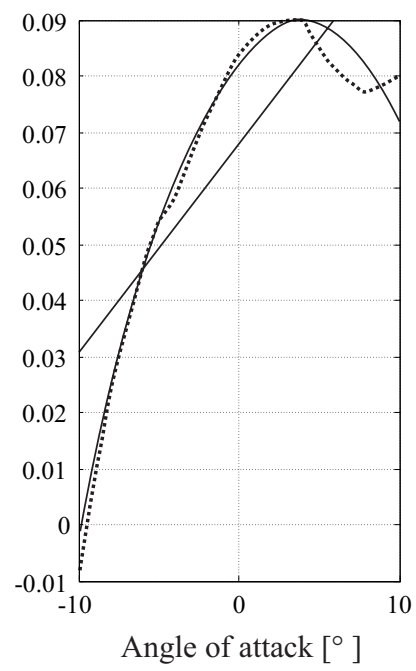

Fig. 2. Linear and quadratic approximation of the drag coefficient (Viaduct of Millau)

For the sake of simplicity in the notations, let us write $f_{6}, f_{7}$ and $f_{8}$ the non dimensional forces defined by relations (6) to (8) respectively. Concerning the linear loading (Equ. 8), the calculus of probabilities shows that the mean and variance are expressed by:

$$
\begin{aligned}
& \mu_{f_{8}}=c_{0} \\
& \sigma_{f_{8}}^{2}=4 c_{0}^{2} I_{u}^{2}+c_{1}^{2} I_{v}^{2}
\end{aligned}
$$

where $I_{u}$ and $I_{v}$ are the turbulence intensities (ratio of standard deviation to mean wind velocity). For the considered drag coefficient, Figure 3 represents, in the first column, the statistical moments obtained with the linear loading. The statistical quantities are represented as a function of wind intensities. Zero values for the third (skewness coefficient) and fourth (excess coefficient) moments show again the Gaussianity of this particular loading.

Equation (7) represents a kind of non linear loading where the non linearity comes from relations (3) only, and thus not from the non linearity of the aerodynamic coefficient. This kind of non linearity has already been studied widely $([8,9,10])$, at least in a 1-D turbulence field. In such a field, the transverse turbulence intensity is equal to zero and the comparison should be made between results reported on the vertical axis only $\left(I_{v}=0\right)$. The conclusions of previous researches were these: almost no influence of the non linearity on the mean (from 0.083 to 0.085), nor on the variance. However, the loading is now non Gaussian and the main difference between loadings (8) and (7) results in the third and fourth order statistical moments.

This second column of graphs in Figure 3 has been drawn from the same kind analytical relation as Equ. (10) but derived from loading (7) and not (6) anymore. They are reported in annex.

When the non linearity of the aerodynamic coefficient is also taken into account (Equ. 6), such an analytical relations for the statistical moments of the loading can also be established. They are also given in annex and are represented in the third column of Fig. 3.

It can be observed that:

- The non linearity of the aerodynamic coefficient affects significantly the mean force. A curvature to the bottom $\left(c_{2}<0\right)$ tends to reduce the mean value whereas a curvature to the top tends to increase it. This point is rather important since extreme values used for the final design are expressed by reference to mean variables, e.g. displacements, stresses, etc. In the particular case of the drag coefficient chosen for this paper, a significant decrease in the mean value can be observed. 
Equ. (8)

Equ. (7)

Equ. (6)
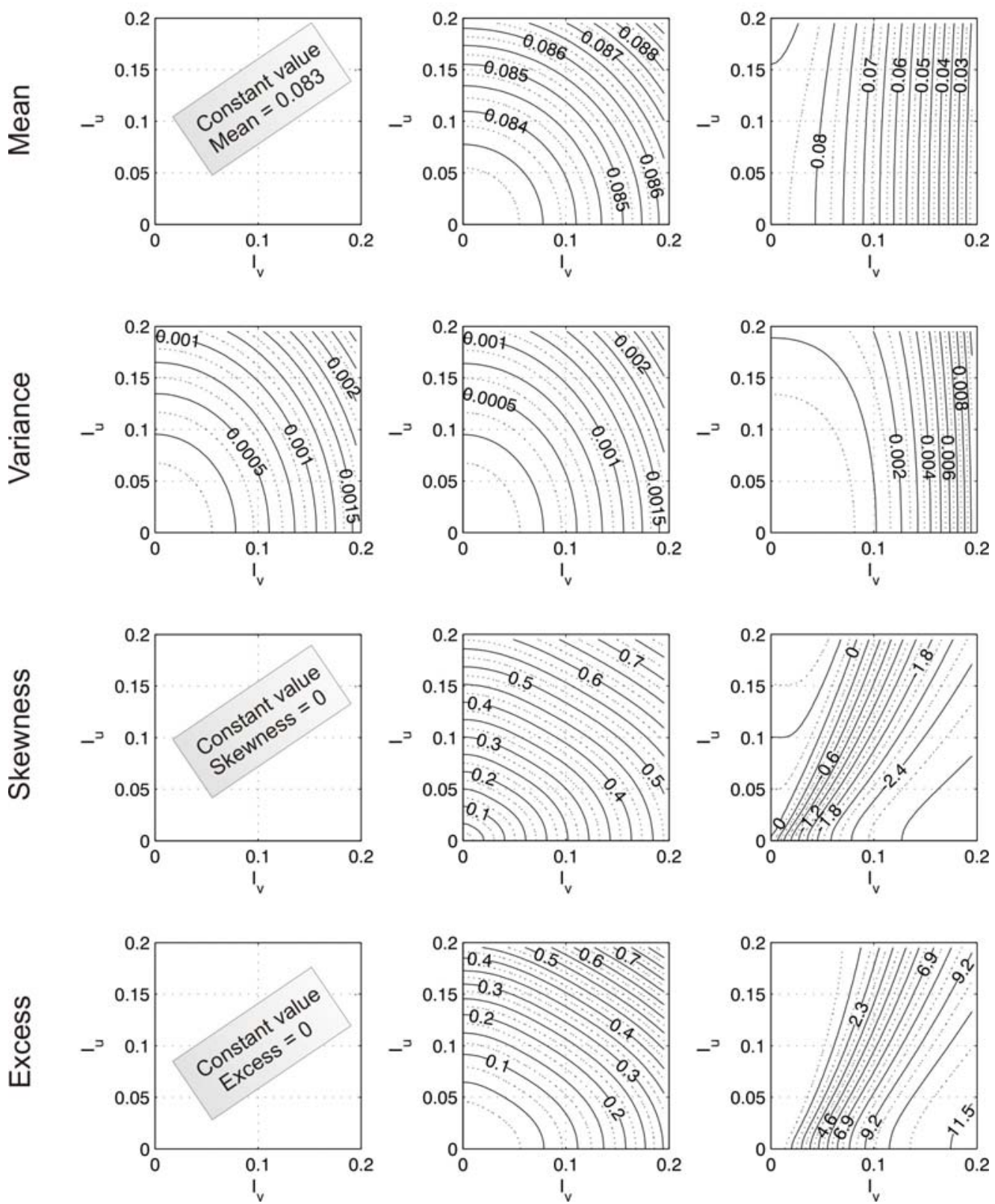

Fig. 3. Statistical characteristics of the loading obtained for three different loadings (Equ. 6 to 8). Application to the drag coefficient of the Viaduct of Millau

- Concerning the second order moments, the non linearity of the aerodynamic coefficient can also affect significantly the variance of the force. The difference arises actually because of the transverse intensity. It can indeed be seen that Equs. 7 and 8 provide the same values on the vertical axis. Despite this perfect coincidence on the vertical axis, the importance of the non linearity can be important and the transverse turbulence mustn't necessarily be important to have a significant effect: a $10 \%$ transverse turbulence gives in this case a standard deviation which is twice larger! Because of the hypothesis of uncoupling in the turbulence components, it 
can be proved that the non linearity of the aerodynamic coefficient leads always to an increase in the variance of the force (no matter the sign of $c_{2}$ ).

- Concerning third and fourth statistical moments, the non linearity of the aerodynamic coefficient is also very important. Contrarily to loading 7 which can lead to positive skewness coefficients (from 0 to 0.8 ) only, loading 8 can lead to positive or negative skewness. The values that can be reached are much more important (in absolute value). This could be drastically important in terms of extreme values. The existence of a limit between positively and negatively skewed forces is quite interesting. A clever designer could take advantage of this property in order to design a bridge deck section such that the on-site wind turbulences $\left(I_{u}, I_{v}\right)$ lies on this limit. In this case, the loading is not skewed and the estimation of extreme values is much easier (because the hypothesis of Gaussian process could eventually be done).

As a conclusion, in any case, it can be said that the non linearity of the aerodynamic coefficient is important. In case of 1-D flow (no transverse turbulence; one must read results on the vertical axis), graphs of columns 2 and 3 correspond for moments 2,3 and 4 . This explains why former researches in 1-D flows did not focus on the influence of the non linearity of aerodynamic coefficients. It has been shown however that this point is important in case of 2-D flows, at least concerning the statistical characteristics of the loading.

\subsection{Illustration by Monte Carlo simulation}

To check the validity of the analytical relations derived in the previous paragraph, one could realize Monte Carlo simulations of the wind forces with each of Equs. 6 to 8. Whenever an analytical approach is available, it is desirable not to use any Monte Carlo simulation, simply because the establishment of the influence patterns of Fig. 3 could take a long time to be computed. Since these simulation are used here for a checking, it will be supposed that the turbulence intensity is $15 \%$ for each turbulence component $\left(I_{u}=0.15\right.$ and $I_{v}=0.15$ ).

In order to compare the wind models, a long Gaussian sample ( $N=500.000$ points) has been generated for each component of the wind turbulence. Then the non-dimensional expressions of the three loadings (Equs. 6 to 8) have been used in order to establish histograms of the non-dimensional drag force (Fig. 4).

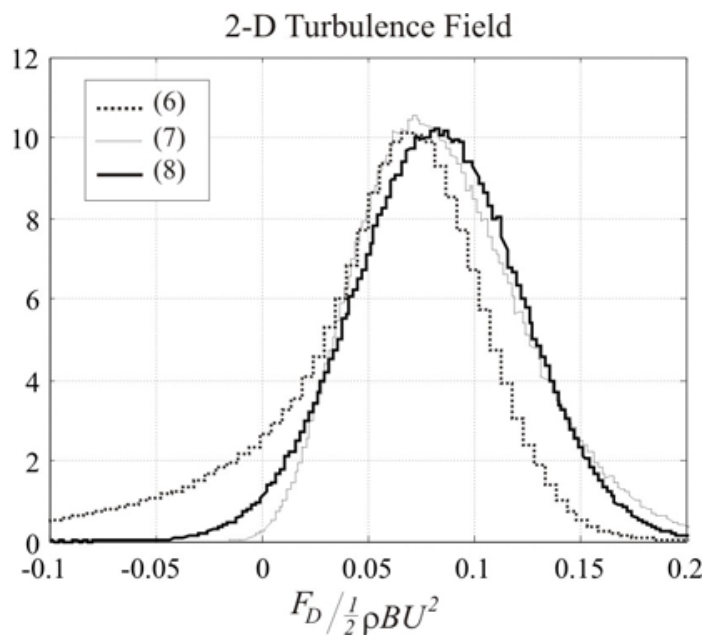

Fig. 4. Probability density function of the non dimensional drag force obtained with different wind models 
Vincent Denoël, Hervé Degée

\begin{tabular}{ccccccccc}
\hline Equ. & \multicolumn{2}{c}{ Mean } & \multicolumn{2}{c}{ Std. Dev. } & \multicolumn{2}{c}{ Skewness } & \multicolumn{2}{c}{ Excess } \\
\hline & Analytic & MC & Analytic & MC & Analytic & MC & Analytic & MC \\
$(6)$ & 0.0485 & 0.0484 & 0.0649 & 0.0649 & -2.0565 & -2.0669 & 7.8020 & 7.8811 \\
$(7)$ & 0.0867 & 0.0867 & 0.0397 & 0.0397 & 0.6147 & 0.6177 & 0.5125 & 0.5336 \\
$(8)$ & 0.0830 & 0.0829 & 0.0392 & 0.0392 & 0 & 0.0007 & 0 & 0.0027 \\
\hline
\end{tabular}

Table 2. Statistical characteristics of the forces computed by analytical relations and Monte Carlo simulation.

The thick line represents the linear model leading to a Gaussian process. The thin line is the first non linear loading model. Based on analytical developments of the previous paragraph, we had previously concluded to a slight influence on the mean and variance as well as a positive skewness coefficient. These comments can again be observed by looking at the probability density function. Concerning the last loading, including the non linearity of the aerodynamic coefficient, the probability density function exhibits a much more evident non Gaussian shape. The decrease in the mean value, the increase in the variance and the negatively skewed loading can again be observed.

For both analytical and Monte Carlo simulation methods, Table 2 summarises the statistical characteristics (up to the fourth order) obtained for each loading. The very good accordance between results obtained with both methods proves the validity of the analytical relations.

Figure 4 is also interesting because the representation of the non Gaussian probability density functions is a good illustration to show that the statistics of extreme values are completely different. Exactly as extreme values of Gaussian process can be expressed by the product of the standard deviation and a peak factor [7], some mathematical models allow to represent the extreme value statistics of non Gaussian processes a functions of skewness and excess coefficients. The developments presented in this paper are thus exactly the necessary tools to estimate the statistics of extreme values, which, in turn, are needed to realize the final design of a structure.

\section{SPECTRAL ANALYSIS OF STRUCTURES SUBJECTED TO RANDOM LOADING}

Even if it is important to describe the loading as precisely as possible, designers are however mainly interested in determining the structural displacements, internal forces and stresses. In this section, we will give a schematic summary of the procedure to follow in order to establish the response of a structure subjected to a random dynamic loading.

\subsection{Second order characteristics}

The second order characteristics of a random loading are represented by its power spectral density (PSD). The PSD of the applied force represents the distribution in the frequency domain of the variance of the process. The frequency content of the response, i.e. its PSD (see Fig. 5), can be obtained by multiplying the PSD of the force by the squared transfer function of the single degree of freedom system. The surface under the resulting function (function of the frequency) is equal to the variance of the response.

\subsection{Third order characteristics}

At the third order, the reasoning is identical but somewhat more complex since one dimension is added. The third order statistical characteristics of the loading are represented by its bispectrum. This function represents the distribution, in a 2-D frequency space, of the third order statistical moment of the loading. On the second line of Fig. 6, an example of typical 
wind force bispectrum is given. Exactly as the transfer function $\left(2^{\text {nd }}\right.$ order $)$ represents the way a frequency content must be filtered, at the third order, the Volterra kernel represents the way the bispectrum of the force must be filtered in order to obtain the bispectrum of the response.

In other words, exactly as it was done at the second order, a simple multiplication of the two lower left functions gives the bispectrum of the displacements. Then, the computation of the volume under this surface gives the third order moment, which in turn, gives an estimation of the skewness coefficient.

\subsection{Fourth order characteristics}

The fourth order characteristics of the response can be established following an identical procedure but working now with a supplementary dimension. This makes the graphical representation quite complicated. Anyway, it must be understood that the trispectrum (distribution of the fourth order moment in a 3-D frequency space) of the response can be obtained by multiplying the trispectrum of the force by a fourth order Volterra kernel.
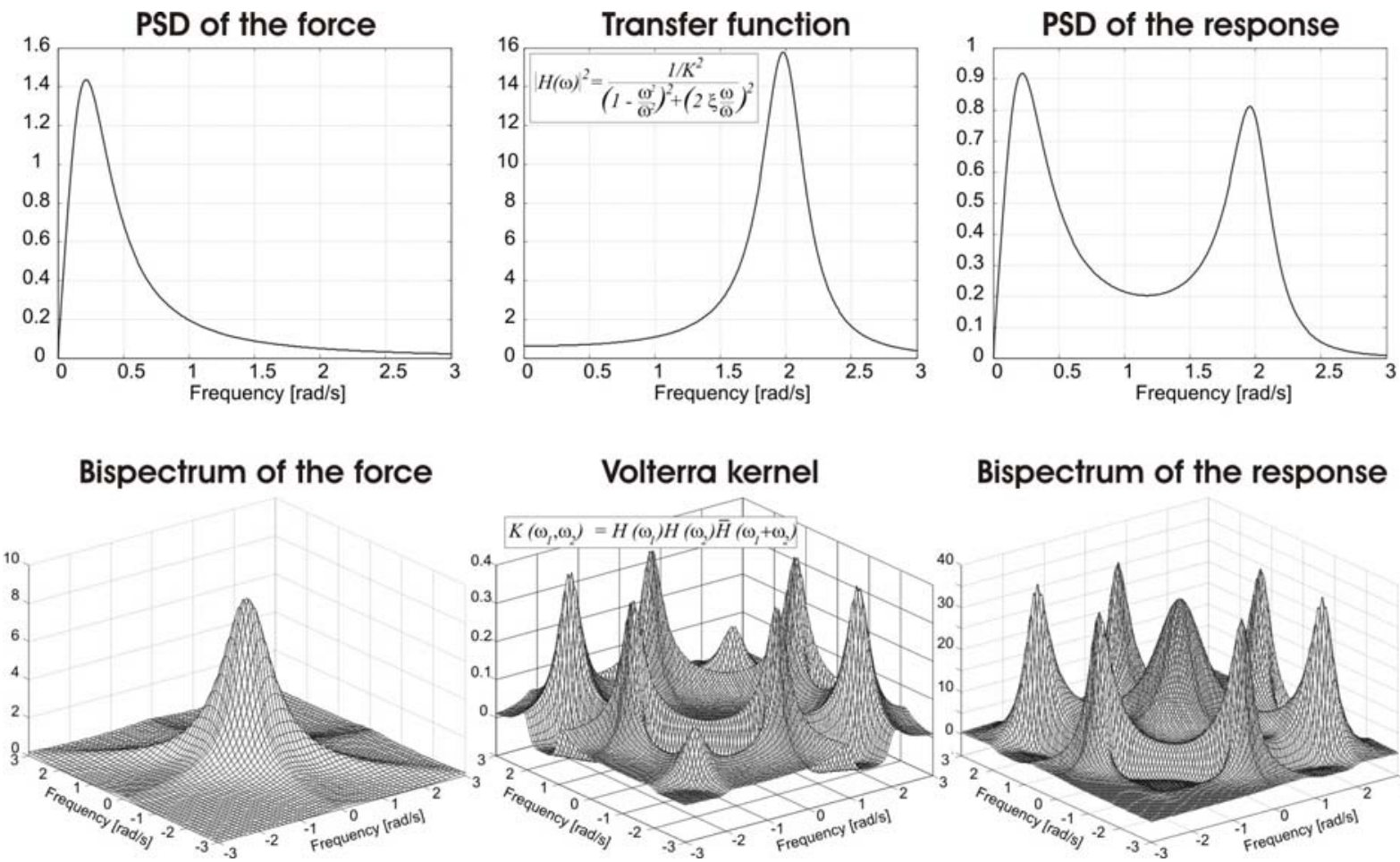

Bispectrum of the response

Fig. 5 Schematic representation of the analysis of a single degree of freedom structure subjected to a random loading $\left(2^{\text {nd }}\right.$ and $3^{\text {rd }}$ orders $)$

\section{SIMPLE ESTIMATION OF THE IMPORTANCE OF THE NON LINEARITY OF AN AERODYNAMIC COEFFICIENT}

\subsection{Method : objectives and limitations}

Some finite element codes allow analyzing the non Gaussian response of large FE models, like for instance a 3-D bridge with various non linear aerodynamic coefficients, immerged in a 3-D turbulence flow. It is known that the analysis at the second order is already timeconsuming when the rigorous approach presented in the previous section has to be applied. This observation is again strengthened at the third and fourth orders since the integration has to be performed on 2-D and 3-D frequency spaces. At the very first steps of a new design pro- 
ject, it is unthinkable to run such a complex and complete analysis. It is thus important to be able to estimate, in a very simple manner, the order of magnitude of the main quantities.

In the buffeting analysis of a bridge, the deck is very often responsible for the most important part of the loading. It is thus essential to understand clearly how the forces are generated on the bridge deck. As a particular case, the non linearity of the aerodynamic coefficients of this section should be taken into account. Instead of realizing a complete coupled analysis, we propose a very simple procedure for assessing the influence of the non linearity of aerodynamic coefficients on the structural displacement.

Based on the hypothesis of uncoupled motions in vertical and horizontal bending and torsion, each aerodynamic coefficient could be used separately to study the response of a single degree of freedom system. The comparison of responses obtained with loadings 6 and 7 gives a qualification of the aerodynamic coefficient and indicates whether a more particular attention has to be dedicated to this phenomenon. This sort of qualification of the aerodynamic coefficient is interesting because:

- it replaces a MDOF system by a SDOF system and is hence very easy to apply;

- it can account for the real frequency content of the turbulence components $u(t)$ and $v(t)$ (von Kármán, EC1, Davenport, Harris, ...);

- the operation can be rapidly repeated for any eigen frequency, damping coefficient or wind intensities. This gives thus an interesting mapping that can be used in further developments of the design since, at this stage, the definitive natural frequencies are generally not fixed yet. This kind of mapping can thus show the main tendencies: "what happens in terms of extreme values if the eigen frequency is increased? What is the influence of the damping ratio?" etc.

\subsection{Example}

This method of qualification of aerodynamic coefficients will be applied to the drag coefficient of the Viaduct of Millau. In order to realize the developments in closed form, we will suppose that the power spectral densities of the turbulence components are given by:

$$
S_{u}(\omega)=\frac{\alpha \sigma_{u}^{2}}{\pi\left(\alpha^{2}+\omega^{2}\right)} ; \quad S_{v}(\omega)=\frac{\alpha \sigma_{v}^{2}}{\pi\left(\alpha^{2}+\omega^{2}\right)}
$$

where $\alpha$ is a shape factor (units: $\mathrm{rad} / \mathrm{s}$ ). It has been shown [5] that this shape of PSD is close to practical turbulence PSD’s (von Kármán, Davenport, Eurocode 1...) which can be well reproduced by an appropriate choice of parameter $\alpha$, generally resulting in $\alpha \in[0.2$ $\mathrm{rad} / \mathrm{s} ; 0.5 \mathrm{rad} / \mathrm{s}]$.

In order to estimate the effect of the non linearity of the aerodynamic coefficient, loadings (6) and (7) will be applied to a single degree of freedom system with a unit mass $(M=1)$ :

$$
\ddot{x}(t)+2 \xi \varpi \dot{x}(t)+\varpi^{2} x(t)=\frac{F_{D}(t)}{\frac{1}{2} \rho B U^{2}}
$$

where $\varpi$ and $\xi$ represent respectively the circular eigen frequency and the damping ratio (including the aerodynamic damping). 
Equ. (7)

Equ. (6)
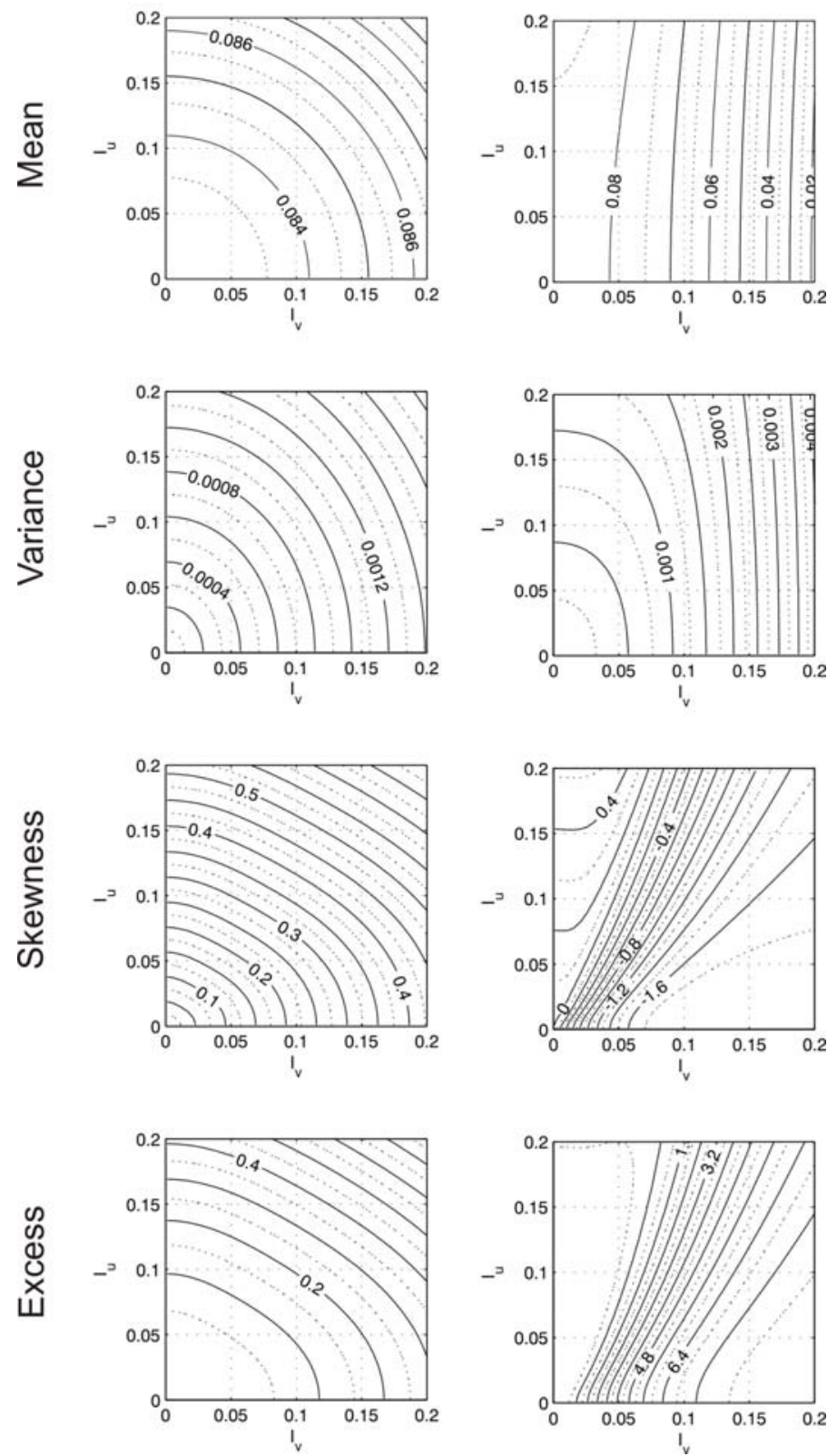

Figure 6. Statistical characteristics of the response obtained for two different loadings (Equ. 6 to 7). Application to the drag coefficient of the Viaduct of Millau - $\varpi=6 \mathrm{rad} / \mathrm{s} ; \xi=0.03, \alpha=0.2 \mathrm{rad} / \mathrm{s}$

The mean displacement of the system is obtained by dividing the mean applied force by the stiffness, i.e. the squared circular frequency. The ratio between the displacements computed with and without the aerodynamic non linearity is thus equal to the corresponding ratio estimated on forces. The first line of figure 6 represents the mean non dimensional displacement as a function of wind intensities. It is exactly the same as the first line of figure 3.

At the first order, the influence of the non linearity of aerodynamic coefficients is independent of the dynamic characteristics of the system ( $\varpi$ and $\xi$ ). For large intensity levels, the curvature of the aerodynamic coefficient (towards the bottom) is so important that it can even decrease the mean value of the response to zero. 
At the second order, the power spectral density of the applied force must be established. Thanks to the simple analytical expression of the PSD's of the turbulence components, this expression is rather simple. It is given in the annex. The application of the procedure summarized in the previous section gives successively analytical expressions for the PSD of the displacement and finally for its standard deviation. This last expression is more complex and is not given in the paper. It is however represented at figure 6, second line, where the influence of the non linearity of the aerodynamic coefficient can again be observed. Figure 6 represents the statistical moments of one particular system ( $\varpi=6 \mathrm{rad} / \mathrm{s} ; \xi=0.03$ ) but it is obvious that any other SDOF system could be used. The same kind of conclusions as those drawn out from the forces can be established: the standard deviation obtained with the quadratic aerodynamic coefficient (Equ. 6) is always larger (for any SDOF and for any aerodynamic coefficient) than the one obtained with the linear coefficient (Equ. 7). This is a consequence of the hypothesis of uncorrelated turbulence components.

It could be interesting to compute the same mapping with other structural characteristics ( $\varpi$ and $\xi$ ). For a very stiff or highly damped structure, the response would be quasi-static only and results presented in figure 6 would correspond closely to those of figure 3 . For more flexible and less damped structure, the dynamic component modifies slightly the pattern. This explains why corresponding graphs of figures 3 and 6 are so similar. This point leads also to this important statement: graphs of figure 3 are very easy to be obtained; they should be computed for any aerodynamic coefficient and then eventually used at the first step of a design, for "stiff” eigen modes.

Computations at the third order become hard to undertake in closed forms. The expression of the bispectrum of the loading can however be established. The bispectrum of the response is obtained by multiplying this quantity by the second Volterra kernel. Finally, an analytical expression of the third order moment can be obtained.

The resulting skewness coefficient is represented in the third line of Fig. 5. A comparison of this graph with the corresponding one established for the loading shows (resp. for Equs 6 and 7) that the skewness coefficient of the response is smaller than the skewness coefficient of the loading. This indicates that the structure acts like a mitigating operator and reduces thus the dissymmetry eventually existing in the loading. Based on the famous decomposition of the response into background and resonant components [1], this observation is a general rule that can be justified by the existence, in the response, of a Gaussian dynamic behaviour and of a non Gaussian quasi-static response. The non Gaussianity of this second contribution to the response comes directly from the non Gaussianity of the loading and is thus mitigated by its dynamic counterpart.

This reasoning leads to the conclusion that the dissymmetry of the response is more important for structures having a mainly quasi-static behaviour.

A negatively (resp. positively) skewed loading results in a negatively (resp. positively) skewed response. The limit between the areas of positively and negatively skewed responses is thus the same as for the loading. This shows again the importance of establishing the very simple graphs of Figure 3.

The same methodology can also be applied at the fourth order. Analytical expressions can be obtained successively for the trispectrum of the loading, the trispectrum of the response, the fourth order statistical moment and finally the excess coefficient. This quantity is represented in the last line of Fig 6. The same kind of conclusions as for the third order is valid: at the fourth order, the structure acts also like a mitigating operator; the excess coefficient of the 
response is always smaller than the excess coefficient of the force, equality arising for very stiff structures.

\section{CONCLUSIONS}

The analysis of a structure subjected to a non Gaussian random loading can be divided into two steps.

The first important step is the characterization of the loading. It is important because the statistical moments of the loading can be estimated very easily, with analytical relations, and can be used to give rough estimations (mainly for stiff structures) of the statistical characteristics of the response. Some information like extreme values of the loading can already show the importance (or not) of the non linearity of the aerodynamic coefficient.

The second step concerns the characterization of the response. Since a complete non Gaussian rigorous analysis is generally not affordable for pre-design of structures, and because the effects of the non linearity of aerodynamic coefficients may have to be taken into account, a simplified procedure has been presented in this paper. It consists in characterizing the influence of the non linearity of aerodynamic coefficients, in the context of a very simple $1 \mathrm{DOF}$, but very close to real conditions. This simplicity and closeness to real conditions are justified by these points:

- natural frequencies, damping ratios and generalized masses are identical;

- the actual frequency content (PSD) of the wind loading can be accounted for;

- in some cases of simple PSD shapes, all equations can be integrated in closed forms, which leads to very fast parametrical studies.

The assessment procedure presented in this paper is so straightforward that it could also be used in further stages of a design project. Indeed, it allowed for instance to state some general comments concerning the influence of the non linearity of the loading:

- due to a mitigating effect of the dynamic component of the response, skewness and excess coefficients of the response are smaller than the corresponding coefficients of the loading;

- it is unnecessary to estimate the non Gaussian characteristics of structures having insignificant quasi-static component; they can be considered as Gaussian.

\section{ACKNOWLEDGMENT}

The authors would like to warmly acknowledge the Belgian National Fund for Scientific Research for having given a financial support to this research.

\section{ANNEX}

The statistical properties of loading (6), up to the fourth order are:

$$
\begin{gathered}
\mu=c_{0}\left(1+I_{u}{ }^{2}\right)+\left(c_{0}+\frac{c_{2}}{2}\right) I_{v}{ }^{2} \\
\sigma^{2}=2 c_{0}^{2} I_{u}{ }^{2}\left(2+I_{u}{ }^{2}\right)+c_{1}{ }^{2} I_{v}{ }^{2}\left(1+I_{u}{ }^{2}\right)+\frac{1}{2}\left(2 c_{0}+c_{2}\right)^{2} I_{v}{ }^{4} \\
k_{3}=8 c_{0}^{3} I_{u}{ }^{4}\left(3+I_{u}{ }^{2}\right)+6 c_{0} c_{1}{ }^{2} I_{u}{ }^{2} I_{v}{ }^{2}\left(2+I_{u}{ }^{2}\right)+3 c_{1}{ }^{2}\left(2 c_{0}+c_{2}\right) I_{v}{ }^{4}\left(1+I_{u}{ }^{2}\right)+\left(2 c_{0}+c_{2}\right)^{3} I_{v}{ }^{6} \\
\gamma_{3}=\frac{k_{3}}{\sigma^{3}}
\end{gathered}
$$




$$
\begin{aligned}
k_{4}= & 48 c_{0}^{4} I_{u}{ }^{6}\left(4+I_{u}{ }^{2}\right)+48 c_{0}^{2} c_{1}^{2} I_{u}{ }^{4} I_{v}{ }^{2}\left(3+I_{u}{ }^{2}\right)+6 c_{1}^{2}\left(c_{1}^{2}+4 c_{0}\left(2 c_{0}+c_{2}\right)\right) I_{u}{ }^{2} I_{v}{ }^{4}\left(2+I_{u}{ }^{2}\right) \\
& +12 c_{1}^{2}\left(2 c_{0}+c_{2}\right)^{2} I_{v}{ }^{6}\left(1+I_{u}{ }^{2}\right)+3\left(2 c_{0}+c_{2}\right)^{4} I_{v}{ }^{8} \\
\gamma_{e}= & \frac{k_{4}}{\sigma^{4}}
\end{aligned}
$$

The statistical properties of loading (7) up to the fourth order are obtained from relations 13 to 16 in which $c_{2}$ is set equal to 0 .

The power spectral density of the applied force (6) is composed of two terms:

$$
S_{f}=\left(4 c_{0}^{2} I_{u}^{2}+c_{1}^{2} I_{v}^{2}\right) \frac{\alpha}{\pi\left(\alpha^{2}+\omega^{2}\right)}+\left(2 c_{0}^{2} I_{u}^{4}+c_{1}^{2} I_{u}^{2} I_{v}^{2}+2\left(c_{0}+\frac{c_{2}}{2}\right)^{2} I_{v}^{4}\right) \frac{2 \alpha}{\pi\left(4 \alpha^{2}+\omega^{2}\right)}
$$

The first term corresponds to the linear terms of loading; the second one comes from the autoconvolutions of the PSD's of the turbulence components and is typical of the non linear loading.

\section{REFERENCES}

[1] Davenport A.G. (1961), "The application of statistical concepts to the wind loading of structures", Proceedings of the Institute of Civil Engineers, Vol 19, 449.

[2] Chen X., Kareem A. (2002), “Advances in modelling of aerodynamic forces on bridge decks”. Journal of Engineering Mechanics, Vol 128-11,1193-1250.

[3] Simiu E., Scanlan R.H (1996), “Wind effects on structures”, John Wiley \& Sons.

[4] Cremona C., Foucriat J. C., “Comportement au vent des ponts”, Presses de l'Ecole Nationale des Ponts et Chaussées (2002).

[5] Denoël V. (2005), “Application des méthodes d'analyse stochastique à l'étude des effets $d u$ vent sur les structures du génie civil”, $\mathrm{PhD}$ thesis, University of Liège, Belgium (in french).

[6] Clough R.W., Penzien J. (1993), Dynamics of Structures, Mc Graw-Hill : Civil Engineering series (second edition).

[7] Gurley K., Tognarelli A., Kareem A. (1997) “Analysis and simulation tools for wind engineering”, Probabilistic Engineering Mechanics, Vol 12(1), 9-31.

[8] Lutes L.D., Hu S.L. (1986) “Non normal stochastic response of linear systems”, Journal of Engineering Mechanics, ASCE, Vol 112, 127-141.

[9] Grigoriu M. (1986), “Response of linear systems to quadratic gaussian excitation”, Journal of Engineering Mechanics ASCE Vol 112(6) 729-744.

[10] Benfratello S., Di Paola M., Spanos P.D. (1998), "Stochastic response of MDOF windexcited structures by means of Volterra series approach”. Journal of Wind Engineering and Industrial Aerodynamics, Vol 74, 1135-1145. 